\title{
Automating the characterisation of beach microplastics through the application of image analyses
}

\author{
Gauci Adam ${ }^{1,}{ }^{*}$, Deidun Alan ${ }^{1}$, Montebello John ${ }^{1}$, Abela John ${ }^{2}$, Galgani Francois ${ }^{3}$
}

${ }^{1}$ Department of Geosciences, Faculty of Science, University of Malta, Msida, Malta

2 Department of Computer Information Systems, Faculty of ICT, University of Malta, Msida, Malta

${ }^{3}$ Station de Corse, IFREMER, Immeuble Agostini- Z.I, Furiani, 20600, Bastia, Corsica, France

*Corresponding author : Adam Gauci, email address : adam.gauci@um.edu.mt

\begin{abstract}
:
Four sandy beaches on the island of Malta in the Central Mediterranean were regularly sampled for Large MicroPlastic (LMP) particles having a diameter between $1 \mathrm{~mm}$ and $5 \mathrm{~mm}$, at stations located at the waterline and $10 \mathrm{~m}$ inshore. The 10975 extracted LMP particles were characterised (dimensions, surface roughness, colour) through unaided visual observation, microscopic analyses, and an algorithm developed within the current study. Two-thirds of the isolated particles were smooth and the majority of these belonged to the grey-white colour category, with a low degree of opaqueness and discolouration, and a high degree of transparency, suggesting that these were dominated by low-density polyethylene LMPs. Conflicting evidence concerning the relative residence time of the isolated LMPs within seawater emerged from the colour and contour roughness determination, although an abundance of primary LMPs (production pellets) within our sample might have been responsible for the low contour roughness results obtained. Roughly six times as many particles were recorded within the inshore sampling stations as the particles recorded at the waterline stations. The developed algorithm performed very well when the dimension and colour parameter values it delivered were compared with those obtained by microscopic analyses.
\end{abstract}

\section{Highlights}

- No universal methodology of high validity for analysing isolated microplastics. Use of image processing techniques to automatically extract parameters for LMPs. Make the process less timeconsuming and removes subjectivity. Samples collected from a number of popular beaches around the island of Malta. Algorithm performed well in determining the dimensions and colour of the LMPs.

Keywords : Image processing, Microplastics, Parameterisation, Monitoring obligations, Marine litter 


\section{Introduction}

The term 'microplastics' first featured in a high-profile, international meeting in June 2014 in Niarobi, Kenya, at the First UN Environment Assembly, which culminated in agreement on a resolution on 'Marine plastic debris and microplastics' (UNEP, 2014). The presence of microplastics in the marine environment is the result of two introduction pathways: as primary microplastics in the form of virgin plastic pellets or powders, and as secondary microplastics through the fragmentation of litter (Primpke et al., 2017).

There are different classification systems for plastics on the basis of size. The definition by Hidalgo et al. (2012) defines Large Microplastics (LMPs) as particles ranging between $500 \mu \mathrm{m}$ and $5 \mathrm{~mm}$ and Small Microplastics (SMPs) as being smaller than $500 \mu \mathrm{m}$. The classification by Galgani et al. (2013) adopted within the EU's Marine Strategy Framework Directive (MSFD) defines the two categories as ranging between $1 \mathrm{~mm}$ and $5 \mathrm{~mm}$, and between $20 \mu \mathrm{m}$ and $1 \mathrm{~mm}$, respectively. Despite such discrepancies in the definition of different microplastic categories, there is widespread recognition that monitoring of MPs is crucial due to the possible uptake by organisms at low trophic levels (Setälä et al., 2014). So much so that one of the indicators currently entrenched for the MSFD's Descriptor 10 (Marine Litter) refers to trends in the amount, distribution, and where possible, composition of micro-particles (10.1.3). Besides the MSFD, the only source of monitoring guidelines for microplastics along the shoreline is the DeFishGear project (http://www.defishgear.net/media-items/publications)

Despite this consensus in recognising the importance of microplastics monitoring protocols, there is no universal methodology of high validity for analysing isolated particles. In fact, the simplest analysis protocol involves examination with the naked eye or with a microscope, without further analyses. However, this results in error margins as high as $70 \%$ when the same samples were re-analysed using spectroscopic techniques (Loder et al., 2015). This is mainly due to human observer interpretation bias which is inevitable in such visual analysis especially when determining subjective attributes such as colour (O'Neill and Smith, 2014). One example, for instance, is the assigned colour. Different observers may assign different colours when carrying out the analysis under different prevailing lighting conditions. There is, therefore, much scope for the formulation of an alternative microplastics analysis technique. Spectroscopic analysis (FT-IR, near-IR, Raman and, most recently, ATR FT-IR) is not widely available (mainly due to cost), and some particle attributes which can shed light on the dynamics of the same particles in the marine environment (e.g. surface roughness), cannot be quantified simply through visual examination. Other analytical techniques, besides spectroscopy, which are deployed for the characterisation of LMPs, including Focal-Plane Array (FPA)-based imaging, are time-consuming.

Galgani et al. (2013) categorise the estimated costs necessary for the monitoring of microplastics on beaches. The costs necessary within the microplastics 'analysis' step are considered by the same authors as being high (i.e. costing up to 100,000 euros), whilst those for microplastic 'sampling,' and 'processing' are considered by the same authors are being medium (i.e. costing up to 50,000 euros) or even low (costing up to 10,000 euros).

This study investigates the use of image processing techniques to automatically extract the required parameters for LMPs exceeding $1 \mathrm{~mm}$ in diameter. Apart from making the process less time-consuming, an automated method would also remove the subjectivity and allows more accurate spatial and temporal comparisons to be made. The proposed algorithm was tested on a large number of LMP samples collected over several weeks from four different beaches along the Maltese coastline, by running exploratory correlations with LMP attribute parameters quantified by a human observer.

\section{Site Selection and Data Collection}

Microplastic samples were collected from a number of popular beaches around the island of Malta. In particular, Riviera Bay, Golden Bay, Ghadira Bay and Pretty Bay, were sampled. Apart from being distributed along different swathes of the north-western and north-eastern coastline of the island of Malta (Figure 1), these beaches are subjected to different wind and wave exposure conditions by virtue of differences in aspect, fetch, bathymetry, and beach profile. This allows for an exploratory correlation of microplastic density with the meteorological conditions that prevailed on the days preceding sampling. Sampling of each beach was carried out every two weeks between August 2018 and 
November 2018. Each sample was collected early in the morning when no people were present and prior to beach-grooming activities by the authorities. This made sampling from the same location on the beach possible. On all four surveyed beaches, sampling was carried out at three replicates to the waterline $(0 \mathrm{~m})$ and at another three replicates located $10 \mathrm{~m}$ inshore.

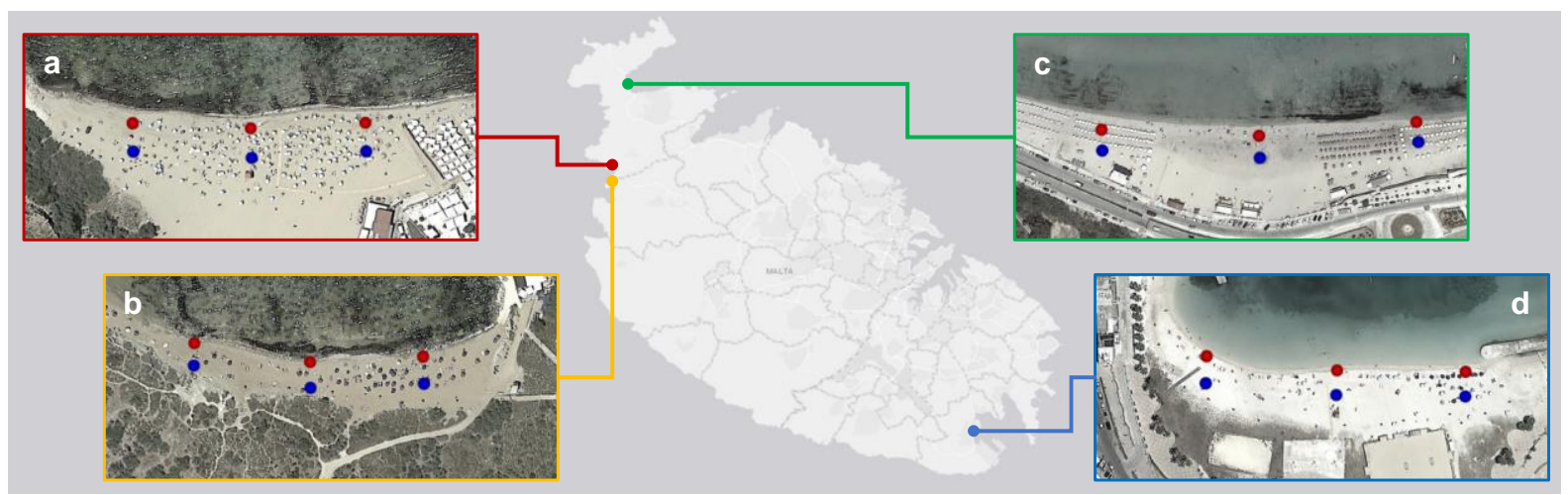

Figure 1: Waterline stations (red dots) and inshore stations (10m towards land as shown by blue dots) that were sampled at Golden Bay (a), Riviera Bay (b),

Ghadira Bay (c), and Pretty Bay (d).

Sand contained within a volume of $50 \mathrm{~cm} \times 50 \mathrm{~cm} \times 10 \mathrm{~cm}$, was sifted through a $5 \mathrm{~mm}$-sieve in order to collect LMP particles have a diameter which exceeded $1 \mathrm{~mm}$. A custom-built wooden frame holding the mesh ensured that a constant volume was sieved every time. At each site, GPS coordinates were recorded so as to ensure concomitant sampling on the same beach on different occasions. A small shovel was used to scoop the top layer of sand in each quadrat. This was passed through the sieve and the process was repeated until all sand was filtered. Debris above $1 \mathrm{~mm}$ was stored in a gauze and labelled accordingly. Due to the hot weather conditions experienced during the summer months, the extracts were stored in a fridge until processed in the lab. This reduced the risk of a variation over time in the LMPs' dimensions, roughness, and colour. Each beach was sampled eight times in total and each sampling session resulted in the collection from six different stations (three replications from two different locations on each beach). Therefore, over eight-weeks, samples were collected from fortyeight individual stations, amounting to a total of one hundred and ninety-two stations for the four beaches combined.

In the lab, separation of the LMPs from other debris was carried out through the use of a high-density saline solution. In particular, a calcium chloride $\left(\mathrm{CaCl}_{2}\right)$ based solution facilitated the separate of LMPs from natural sediment that might not have been filtered through sieving. When placed in this cheap, non-toxic, and zero health-risk solution, the LMPs floated to the surface and could easily be collected. As suggested by Stole (2015), the remaining contents were still checked for higher-density samples such as those made from PVC, or other plastics that were exposed to biofouling.

The differences between LMP and extra-LMP debris densities was exploited so as to allow for the isolation of the LMPs prior to their storage in glass vials (Hidalgo-Ruz et al., 2012). After calcium chloride was added, the plastic content was scooped from the supernatant inside the beaker. The remaining debris was also visually inspected to make sure that no microplastics were overlooked. Once dry, the samples were ready for further processing. Using metal forceps, all floating LMPs were gently extracted from the solution and allowed to dry, before being placed in separate labelled glass vials. The solution was then filtered to extract any remaining microplastic particles, and this procedure was repeated for the remaining samples.

\section{Proposed Automated Technique}

\subsection{Image Acquisition and Calibration}

Microplastics having a diameter smaller than $1 \mathrm{~mm}$ were put on a petri dish and discarded through microscopic analyses prior to further analyses. To obtain a digital image of the LMPs collected in each 
sample, various data capturing methods and backlight conditions were initially tested. An attempt to capture images through the use of mobile phones and Digital Single Lens Reflex (DSLR) cameras was initially made. However, such techniques require the use of transformation tools in order to project the image so that the particles are always perpendicular to the camera. Since one of the objectives of this study was the collection of very small but accurate length measurements, these image-capturing processes did not prove to be feasible. In addition, processing images captured by a camera requires correction of a large number of degrees of freedom (including angle and lighting conditions). Hence, a robust image-capturing method that can easily and widely be used, was adopted.

Ultimately, it was decided to obtain images of the LMPs through scanning on a flatbed scanner at a high resolution. In particular, data were obtained using an HP Scanjet G3010 with a resolution of 600 dots per inch (dpi). This technique produced images having $5100 \times 7020$ pixels. The saved individual JPEG files had a size of about 1.6MB. Even after considerable zooming, each individual LMP could be identified within the image.

The LMPs collected from each quadrat were randomly positioned on the glass of the scanner and scanned. One of the preliminary steps of the algorithm developed in this study is to identify every LMP as an individual entity. Therefore, for better accuracy, the particles were scattered in a way such that they did not touch each other. Acquisition of the first few scans took considerable time as the manual checking of the distribution of plastics on the scanner is a lenghty process. A uniform scattering pattern of the LMPs on the scanner surface was attempted, also through the use of a fine brush. Following the image collection process, the LMPs were again collected and stored. A sample image of the isolated LMPs is shown in Figure 2.

In order for real length measurements of the LMPs to be obtained, the calibration coefficient to convert pixel length measurements into spatial coordinates (having millimetres as units) had to be identified. This was inferred by scanning a custom-designed resolution pattern made of boxes with different dimensions at the edges of the scanning area, as shown in Figure 3. Following the digitisation of this ad-hoc image at the same resolution, the number of pixels between each horizontal and vertical pair of black/white intersections was determined. Since the actual length comprised by this number of pixels was known, the dimensions of each pixel could therefore be worked out. A value was computed for each of the four chequered boxes and the average value was determined. This value, quantified as $0.04008 \mathrm{~mm}$, was adopted for all the images obtained using the same scanning device set at the same resolution. If a new scanner model or resolution settings on the same model are used, then the corresponding pixel dimension calibration coefficient has to be re-determined.

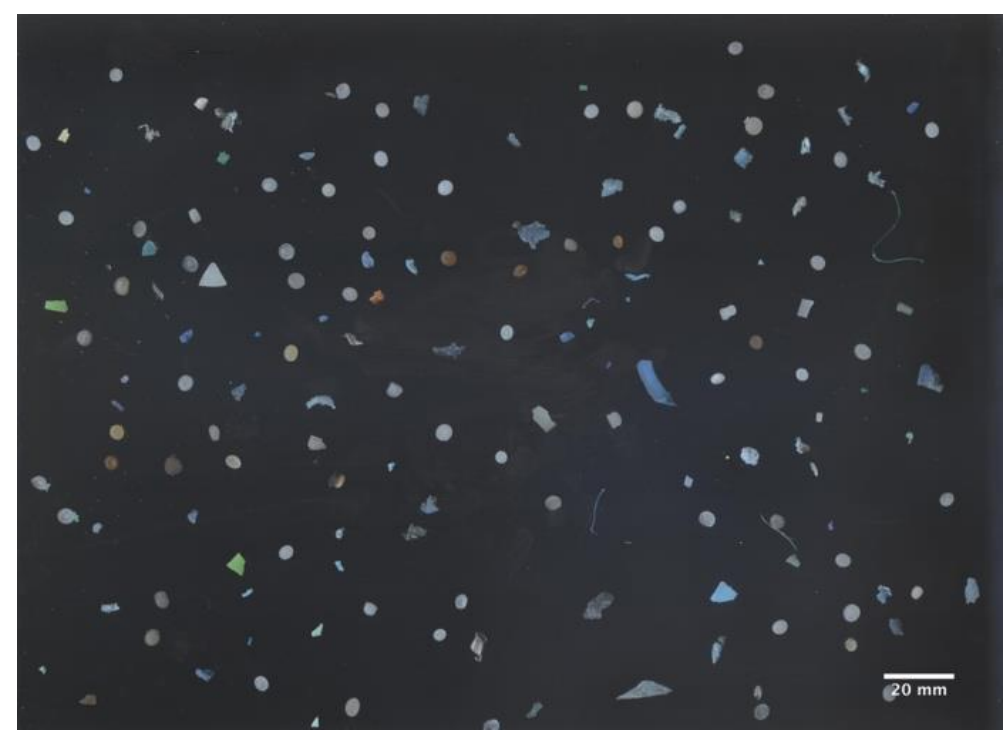

Figure 2: Scanned image of LMPs collected in a quadrat. 


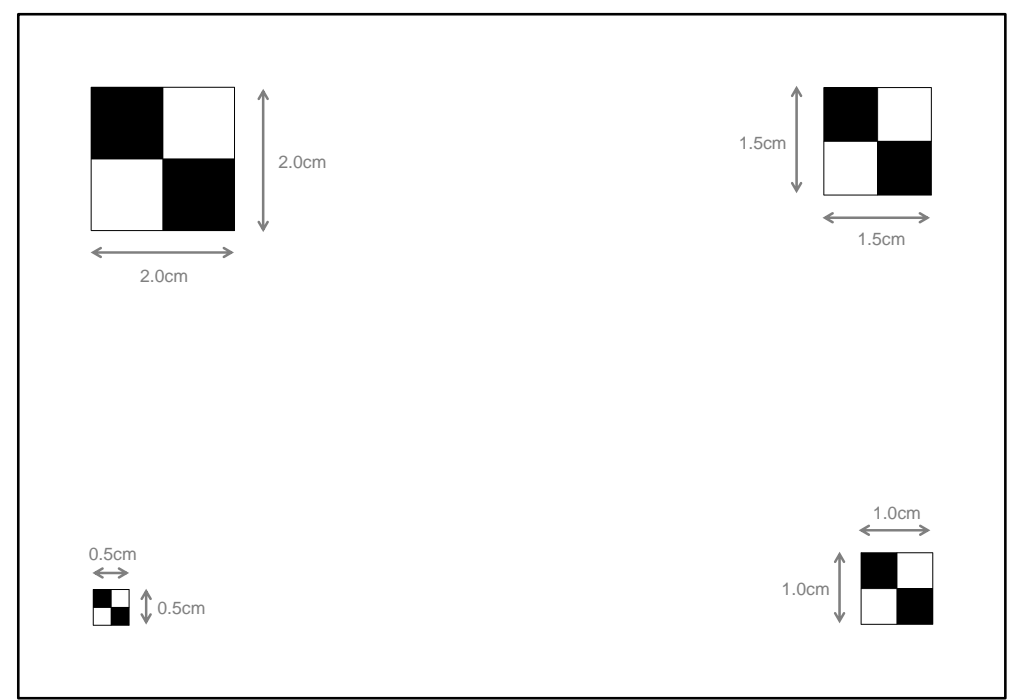

Figure 3: Resolution pattern used to

determine calibration coefficient.

\subsection{Implementation Details}

Following the acquisition of a number of test images, the corresponding algorithm was developed and fine-tuned using Matlab by MathWorks. As demonstrated by Gauci et al. (2018), algorithms that work on natural images often attempt to augment the colour information by projecting the RGB (Red, Green, and Blue) data onto the LAB model. In such cases, the $L$ channel would represent the luminance, the $A$ channel would show the variation from green to red, while the $B$ channel would store the variation from blue to yellow. This approach allows the separation of the luminosity (that may be different across images), from the colour information. In this work, all images were scanned by the same hardware and the dataset is not expected to have variations in brightness. Therefore, images were processed individually in RGB space to avoid unnecessary computation and shorten the required processing time. Each scanned RGB image was initially converted to grayscale through the standard weighted sum approach, that is, by taking $29.89 \%$ of the red channel, $58.70 \%$ of the green channel, and $11.40 \%$ of the blue channel. The resulting 8-bit image with pixel values ranging from 0 (black) to 255 (white) was normalised (through a division by 255) to obtain a matrix ranging between 0 and 1 . Since the particles were scanned against a black background, an adaptive thresholding method was used to obtain a binary image that represents a mask, with background pixels being designated as ' 0 ' and foreground pixels (that is, plastic particles) being designated as ' 1 '. The adaptive method uses the local mean intensity in the neighbourhood of every pixel to automatically compute the best threshold value. This avoids the need to pre-define a single threshold value and make the algorithm universally applicable to all non-uniform illuminated images. Setting one global value will not make the technique universally applicable on all images. Therefore, the algorithm calculates and applies different thresholds over smaller regions of the image to obtain better results. In this case, the regions were set to $637 \times 877$ pixels which corresponds to twice the size of the original image after being divided by 16 . The result of this processing applied to the image shown in Figure 2 is given in Figure 4. 


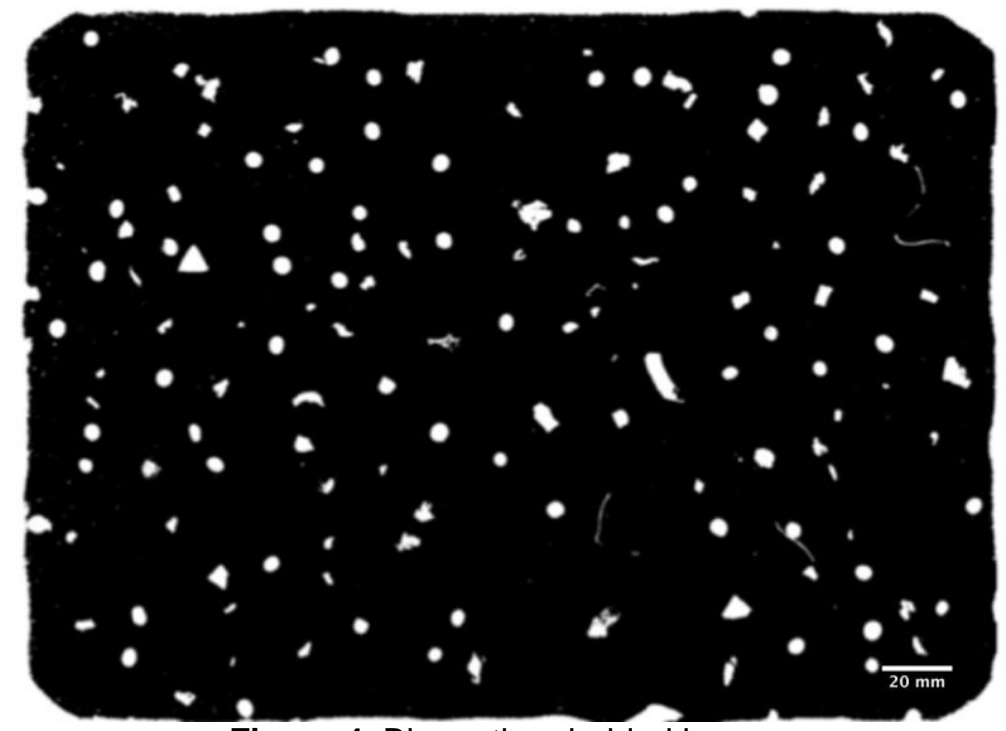

Figure 4: Binary thresholded image.

The next step involved the morphological closing of the binary image through the application of the dilation and erosion operations. During dilation, pixels were added to the boundaries of the detected objects. On the other hand, erosion involved removing pixels from the object boundaries. Such processes are useful for filling small holes in the binary image while preserving the shape and size of the detected objects. The addition or removal of pixels from the binary mask is based on a structuring element. In particular, this was taken to be a flat disk with roughly the same size as the microplastics being processed. Morphology processing involves aligning the structuring element with pixels of the image. If at least one pixel of the structuring element coincides with a foreground pixel, then the input pixel is set as a foreground pixel as well. If all pixels within the structuring element are background pixels, then the input pixel is left as a background pixel. During the dilation step, such rules are applied on the background pixels (i.e., pixels with a value of zero in the binary mask). When erosion is carried out, foreground pixels (i.e., pixels with a value of one in the binary mask), are processed. Since microplastics are not hollow particles, this morphological processing allowed dark background pixels that were initially erroneously set inside a plastic element, to be corrected. As can be seen in Figure 5, the resulting image allows the boundaries of each microplastic to be better delineated.
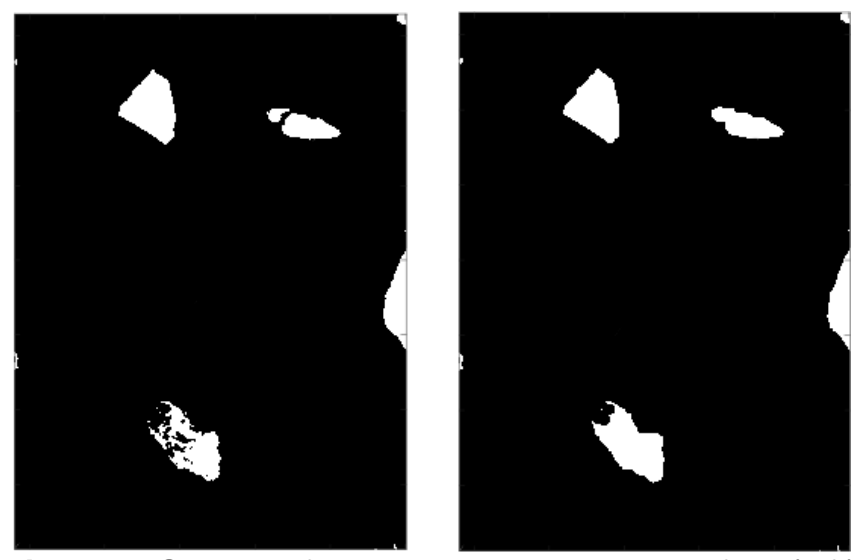

Figure 5: Section of the binary mask image before (left) and after (right) the morphological closing process.

\section{Size Parameter}

The algorithm progressed by identifying the boundaries of objects within the processed binary mask. The perimeter of every foreground (i.e. white) object in the processed image was traced to determine the border. The coordinates of the resulting polygon were extracted and considered to enclose each 
microplastic particle. Very small objects with a boundary definition of less than a few points were ignored. These discarded entities corresponded to dirt or sand particles originally present on the scanner glass surface. For every identified LMP particle, the best ellipsoid was fitted. This was done through a least squares method. The coefficients of the ellipse were adapted until the difference between the boundary of the object and the fit was at a minimum. From the information emerging from this 'harmonisation' exercise, the major and minor axis of the ellipse that corresponded to the dimensions of the detected LMPs were extracted and converted to length measurements through the use of the pixel dimension calibration coefficient as identified previously. In Figure 6, some examples of the detected LMP particles, their identified boundaries (in green), and the corresponding fitted ellipsoids (in red), are provided.

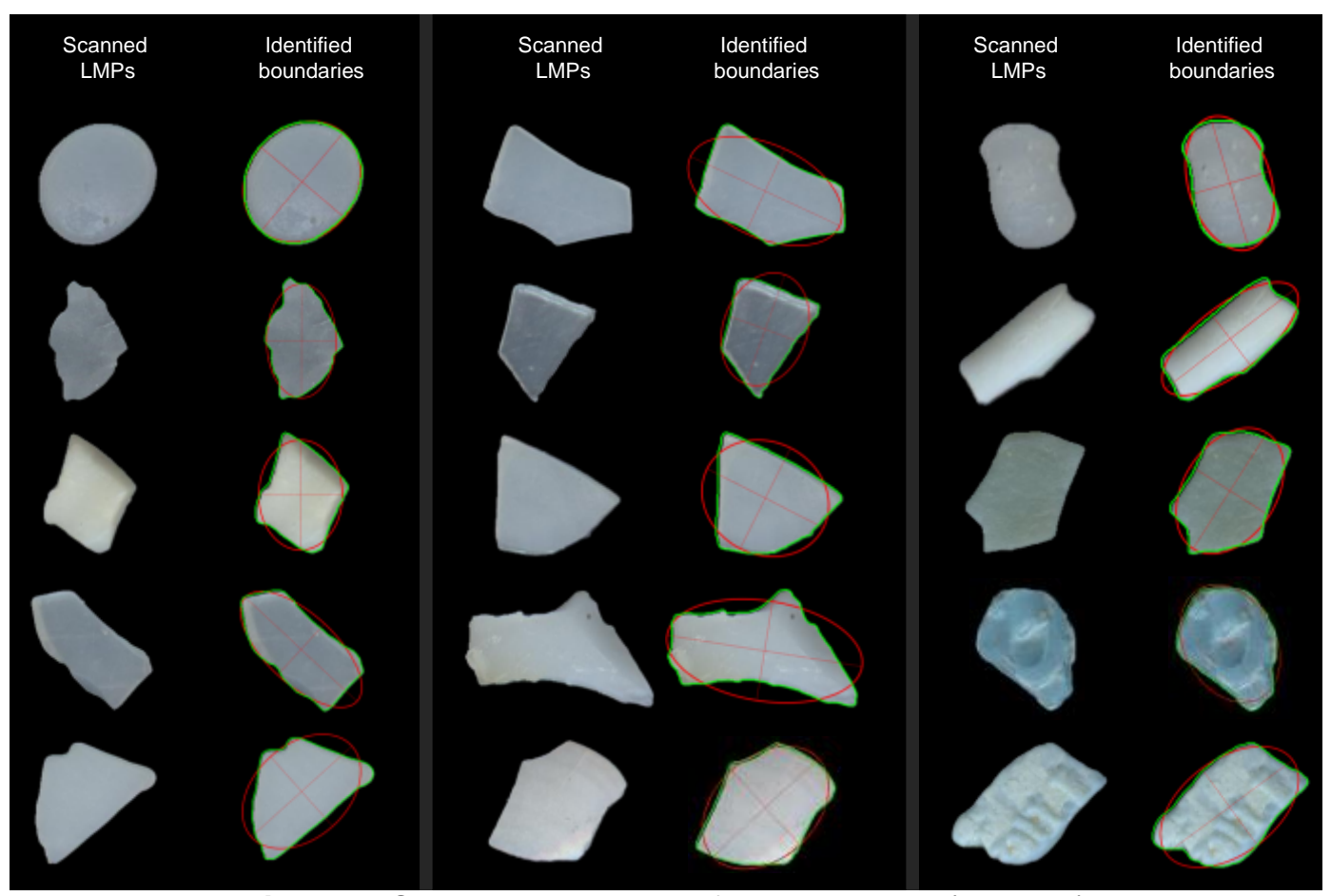

Figure 6: Scanned LMPs, identified boundaries (in green) and the corresponding fitted ellipsoids (in red).

\section{Roughness Parameter}

The difference between the boundary and the best-fitting ellipsoid around each object was used to obtain a measure of roughness. If $B_{\text {area }}$ is taken to represent the total area enclosed by the boundary and $E_{\text {area }}$ is taken to be the area enclosed by the fitted ellipse, then the roughness of the particle $(R)$ is computed as shown in Equation 1. For a perfectly spherical LMP particle, the fitted ellipse will exactly match the corresponding boundary. Therefore, the surface area difference would be minimal and the numerator would have a very small value in this case. On the other hand, the boundary of a non-smooth LMP particle would be very different than the ellipse and the numerator value would be large. Figure 7 visually shows the difference between five different particles that range from smooth ( $R$ value of 0.055$)$ to rough ( $R$ value of 15.892). LMPs corresponding to high roughness values stop having spherical structures and attain more straight edges.

$$
R=\frac{\left|E_{\text {area }}-B_{\text {area }}\right|}{E_{\text {area }}} \quad \text { Equation } 1
$$




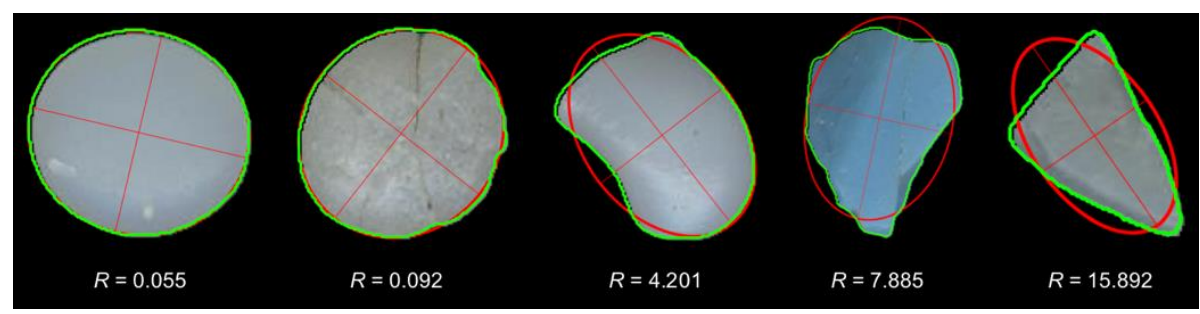

Figure 7: Smooth particle with an $R$ value of 0.005 (left)

and a rough particle with an $R$ value of 0.442 (right).

The accuracy of the computed roughness depends on the output of the morphological process that is applied in the previous step. As discussed in Section 3.2, closing the image will fill small holes in the image caused by dirt, colour discoloration, or shadows. In-turn, this will help to compute a perimeter that will more accurately trace each plastic particle.

\section{Colour Parameter}

The red, green, and blue components of pixels enclosed within each boundary (that is, each detected microplastic particle) were then obtained from the original coloured scan image. The histogram of each colour channel was computed and the mean intensities for each colour $\left(R_{\text {avg }}, G_{\text {avg }}\right.$, and $\left.B_{\text {avg }}\right)$, were obtained. Such values were compared to tuples in a list that stored the information of standard colours. Each LMP was classified as being either black, blue, brown, green, grey, orange, pink, purple, red, white, or yellow. For each recorded colour (defined by its R, G, and B components), the Mean Square Error (MSE) was computed as shown in Equation 2 and the one with the least value was selected.

$$
S(R, G, B)=\sqrt{\left(R_{\text {avg }}-R\right)^{2}+\left(G_{\text {avg }}-G\right)^{2}+\left(B_{\text {avg }}-B\right)^{2}} \quad \text { Equation } 2
$$

\section{Results}

\subsection{Performance evaluation}

With respect to the algorithm validation, Figure 8 gives the LMP particles isolated on the beach of Ghadira. Six of these particles (samples $3,4,8,13,17,18$ ) were used to test the validity of the algorithm's outputs. These samples were chosen to represent different typologies of LMP particles, in terms of shape, dimensions, and colour.

After adopting the standard grid method for LMPs, individual particles were used for a more accurate manual measurement of dimensions through the use of a measurement grid. As shown in Figure 9, individual plastic particles were viewed under a stereomicroscope, which was used to capture micrographs of the same particles, complete with a scale bar.

Following sampling, lab extraction, and image acquisition, the algorithm was run on all isolated LMP samples. The automatically parameterised characterisation of different LMP attributes allowed the rapid collection of results and for statistical analysis to be carried out. A number of different graphical representations allowed a visual comparative analysis to be run.

Table 1 summarises the dimension values as emerging from the three different measurement methodologies applied to the chosen LPM particles. The values in the first column indicate the sample number that is used for reference. The second and third columns present the lengths of the major and minor axis that were read manually from the graph paper. The measurements read from the scale in the images captured by the microscope are shown in columns four and five. These values are considered for ground truthing. Columns six and sever, as well as eight and nine show the number of pixels and the dimensions (in $\mathrm{mm}$ ) computed by the algorithm. The error in the lengths of the major and minor axis is considered to be the difference between the values read manually during microscope analysis and the values produced by the algorithm. These results are shown in columns 10 and 11 . 
The bar graph in Figure 10 shows the distribution of surface roughness values computed for LMP particles isolated from the four different beaches. As demonstrated in Figure 7, smaller values of $R$ indicate smoother samples. Since $66.63 \%$ of the isolated particles (that is, a total of 7133 LMP particles) were classified in the first bin (0 to $5 \%$ ), it can be concluded that the majority of the particles were smooth, that is, they approximate a spherical and perfectly round shape.

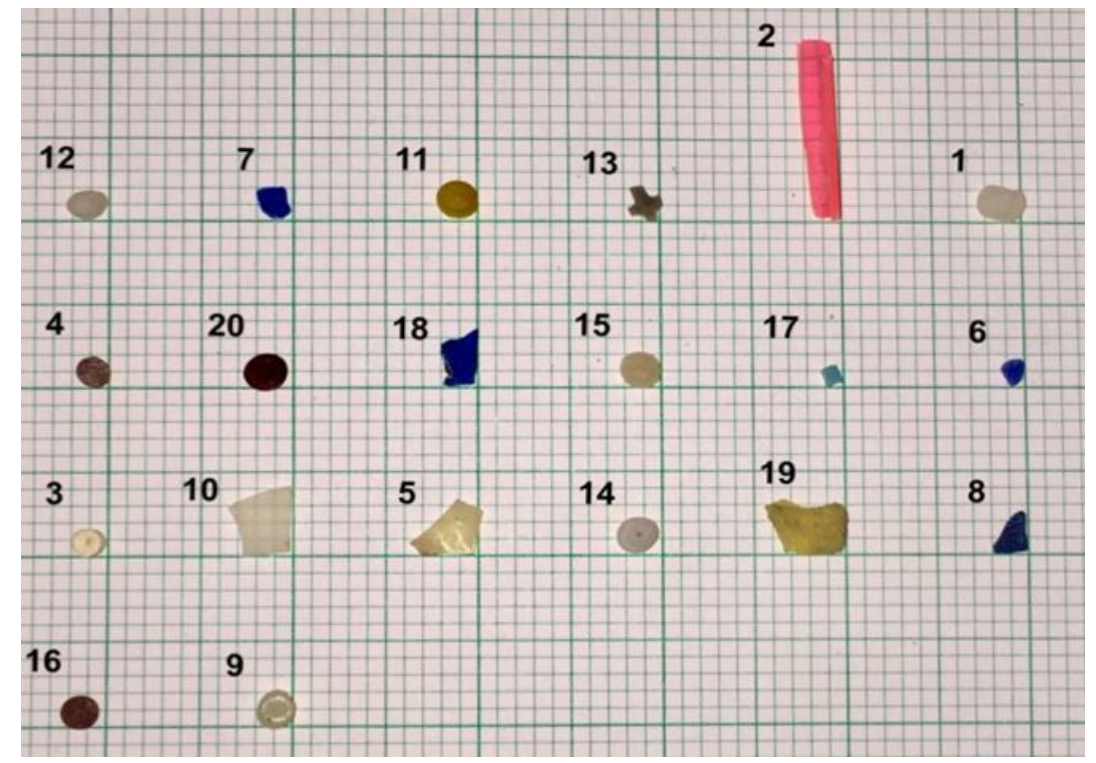

Figure 8: LMPs (and corresponding sample number) extracted from Ghadira placed on a graph paper for manual colour extraction.

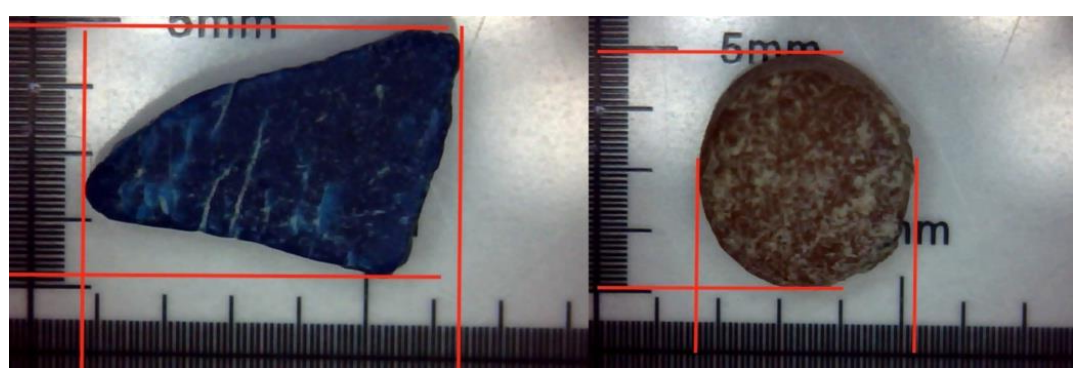

Figure 9: Individual plastics as seen under the microscope, including a scale for measurements.

Table 1: LMP particle dimension values as delivered by the three different measurement techniques, along with the maximum error recorded.

\begin{tabular}{|c|c|c|c|c|c|c|c|c|c|c|}
\hline \multirow[t]{2}{*}{$\begin{array}{c}\text { Sample } \\
\text { No. }\end{array}$} & \multicolumn{2}{|c|}{$\begin{array}{l}\text { Manual grid } \\
\text { measurement } \\
(\mathrm{mm})\end{array}$} & \multicolumn{2}{|c|}{$\begin{array}{c}\text { Microscopic } \\
\text { analyses }(\mathrm{mm})\end{array}$} & \multicolumn{2}{|c|}{$\begin{array}{l}\text { Algorithm output } \\
\text { (no. of pixels) }\end{array}$} & \multicolumn{2}{|c|}{$\begin{array}{l}\text { Algorithm output } \\
(\mathrm{mm})\end{array}$} & \multirow{2}{*}{$\begin{array}{c}\begin{array}{c}\text { Max } \\
\text { error } \\
(\mathrm{mm})\end{array} \\
\begin{array}{c}\text { major } \\
\text { axis }\end{array}\end{array}$} & \multirow{2}{*}{$\begin{array}{c}\begin{array}{c}\text { Max } \\
\text { error } \\
(\mathbf{m m})\end{array} \\
\begin{array}{c}\text { minor } \\
\text { axis }\end{array}\end{array}$} \\
\hline & $\begin{array}{l}\text { major } \\
\text { axis }\end{array}$ & $\begin{array}{l}\text { minor } \\
\text { axis }\end{array}$ & $\begin{array}{l}\text { major } \\
\text { axis }\end{array}$ & $\begin{array}{l}\text { minor } \\
\text { axis }\end{array}$ & $\begin{array}{l}\text { major } \\
\text { axis }\end{array}$ & $\begin{array}{l}\text { minor } \\
\text { axis }\end{array}$ & $\begin{array}{l}\text { major } \\
\text { axis }\end{array}$ & $\begin{array}{l}\text { minor } \\
\text { axis }\end{array}$ & & \\
\hline 3 & 4.0 & 4.0 & 3.3 & 3.3 & 95.08 & 77.72 & 3.98 & 3.20 & 0.68 & 0.10 \\
\hline 4 & 4.0 & 4.0 & 3.5 & 3.8 & $\begin{array}{l}89.77 \\
\end{array}$ & 86.43 & 3.75 & 3.61 & 0.25 & 0.19 \\
\hline 8 & 6.0 & 4.0 & 5.6 & 3.7 & 135.13 & 66.78 & 5.65 & 2.79 & 0.05 & 0.91 \\
\hline 13 & 4.0 & 4.0 & 4.8 & 4.0 & 107.68 & 85.90 & 4.50 & 3.59 & 0.30 & 0.41 \\
\hline 17 & 2.0 & 2.0 & 1.8 & 2.2 & 76.452 & 53.31 & 3.20 & 2.23 & 1.40 & 0.03 \\
\hline 18 & 8.0 & 4.0 & 7.4 & 4.5 & 164.84 & 102.19 & 6.89 & 4.27 & 0.51 & 0.23 \\
\hline
\end{tabular}




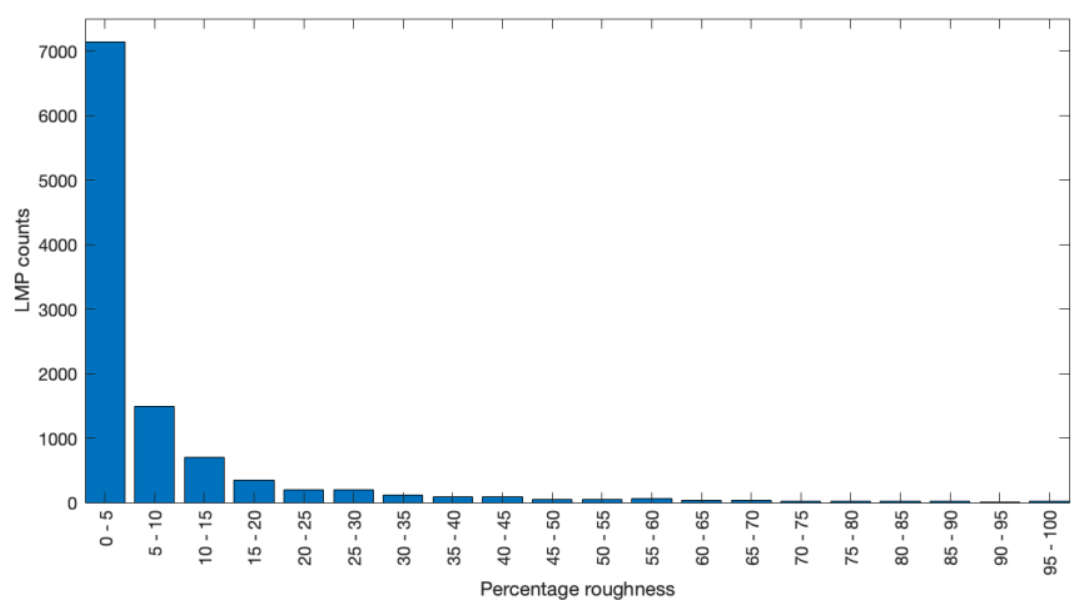

Figure 10: Histogram representing the percentage roughness of different microplastics obtained from the fieldwork, and the results of the image processing to determine individual roughness.

The graph in Figure 11 shows the distribution of the colour parameter of the isolated particles. Most of the particles were of the grey or white category, with a high degree of opaqueness. Only $9.35 \%$ of the samples collected had a specific colour. In general, the developed algorithm detected the same LMP particle colour as that recorded by visual, unaided observation. The distribution of recorded LMP dimensions is presented in Figure 12. Figure 13 presents a comparison of the abundance of LMP particles recorded for each sampled beach at the water line and at $10 \mathrm{~m}$ inshore.

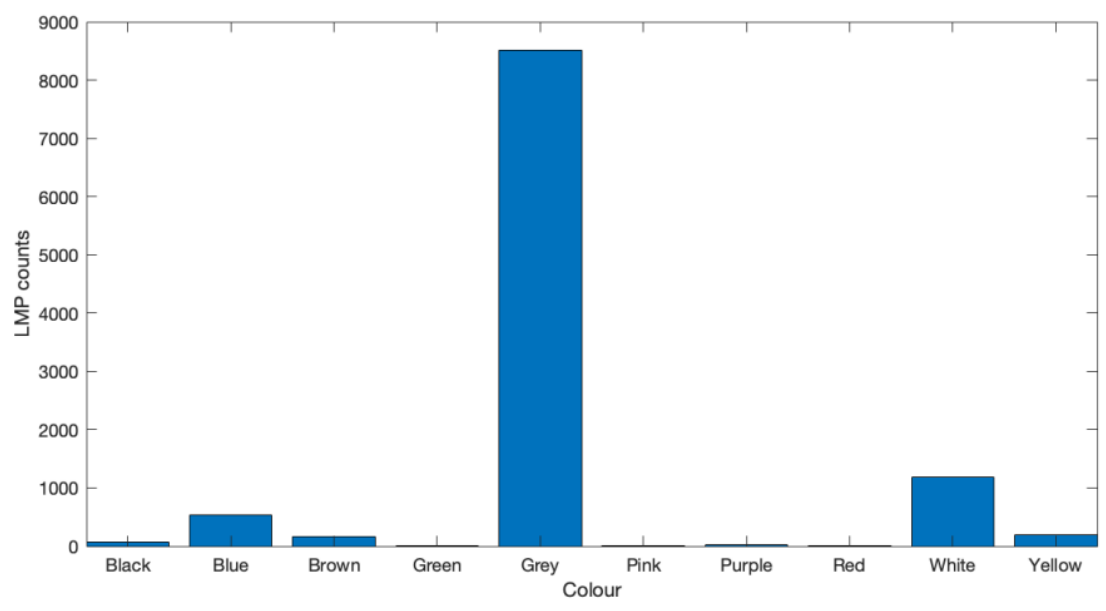

Figure 11: Number of particles per colour category resulting from image processing.

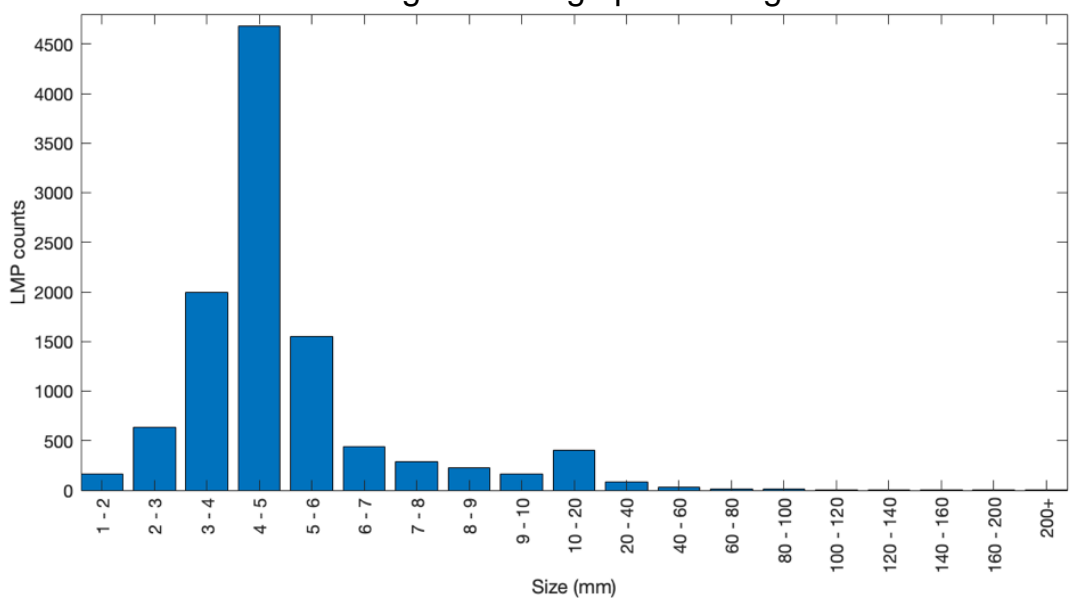


Figure 12: Number of particles by size classes resulting from image processing.

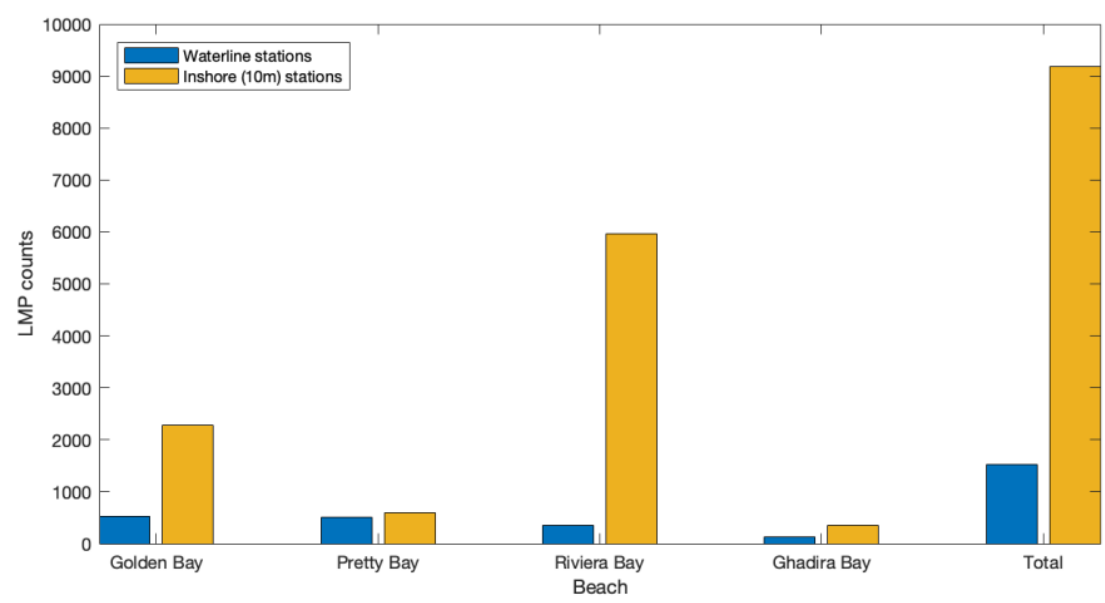

Figure 13: Abundance of LMP particles detected at the different sampling stations on each beach by the image processing method.

\section{Discussion and conclusions}

The beaches with the highest LMP particle counts were found in the current study to be Riviera Bay (6307 pieces) and Golden Bay (2807 pieces). Both have a North-West aspect, which is also the direction of the prevailing wind in the Maltese Islands. Therefore, these two beaches have a profile which is highly-exposed to wind and wave action. The preliminary results from this study suggest that most of the beached microplastics are deposited through hydrodynamic forces, although the contribution from human visitors cannot be completely discounted. The two beaches are, in fact, very popular in summer for bathing purposes. Although Golden Bay (a Blue Flag beach) is regularly cleaned and groomed on a daily basis, Riviera beach is less accessible to motorised beach cleaning facilities. It is not clear, however, whether cleaning activities conducted on Maltese beaches could contribute towards the mitigation of LMP particles on the same beaches given the small dimension of such particles. Similar trends were found in Pretty Bay, both at the waterline and at the inshore sampling stations. In particular, 502 and 600 particles were recorded, respectively. The abundance of LMPs at the inshore sampling station was considerably higher than that recorded at the waterline on the three other surveyed beaches, with a total of 9189 and 1516 LMP particles being recorded on all four beaches at the inshore and waterline sampling stations, respectively. This observation leads us to hypothesize that either (i) sea-borne LMP particles which are eventually beached tend to accumulate and to be transported (putatively through aeolian transport) further inland over time, or (ii) that terrestrial inputs of LMP particles to beaches are important, especially considering that most Maltese beaches are located at the mouth of a valley watercourse (Deidun \& Schembri, 2004).

The algorithm developed through the current study performed reasonably well in determining the dimensions and colour of the limited number of LMP particles subjected to different assessments. No performance evaluation through a corresponding microscopic analyses exercise for LPM particle surface roughness was conducted and thus the algorithm's performance for this parameter could not be assessed. Yet another caveat of the developed algorithm is that it fails to detect 'false positives', i.e. it fails to discriminate between LMPs and non-LMP particles, in that it lacks the compositional analysis element, thus relying on a robust LMP extraction and pre-processing stages. Whilst speeding up the LMP characterisation stages, the developed algorithm still relies on conventional, lengthy LMP extraction techniques. A further valid observation is that the results of the present study are based on image analyses protocols applied to a relatively small cohort of LMPs, despite the LMPs subjected to the analysis being randomly selected. The characterisation of the 'colour' and contour 'roughness/smoothness' through the image analyses protocols described in this study is nonetheless considered useful, particularly as a cost-effective, scoping tool, given that preliminary conclusions on the composition and relative residence time in seawater of LMPs one can infer from the same two attributes. 
A preliminary assessment of the abundance of microplastic particles on Maltese beaches concluded that the same beaches harbour lower microplastic abundance values than other European beaches, with polyethylene, polypropylene, and paint fragments being the most frequently-recorded microplastic typologies on the sampled beaches (Axiak et al., 2017).

The determination of the 'colour' attribute has been used for a preliminary identification of the chemical composition of the most common pellets (Abu-Hilal et al., 2009), with clear and transparent pellets being ascribed to polypropylene and white plastic pellets being ascribed to polyethylene, although more indepth chemical analysis would be necessary to confirm these inferrences. The degree of opaqueness has also been used as a proxy indicator of chemical composition, with opaque colours denoting lowdensity polyethylene and clear-transparent colours denoting ethyl vinyl acetate (Shiber, 1987). Based on these observations by previous workers, the LMPs isolated in the present study were dominated by low-density polyethylene particles.

The 'colour' attribute has also been adopted as a proxy of photo-degradation and residence time at the sea surface, with discolouration/yellowing being indicative of a longer exposure time to seawaters, resulting in polymer oxidation (Endo et al., 2005). The fact that the LMPs extracted in our study generally exhibited a low degree of discolouration might suggest a relatively low residence time in seawater for the same LMPs. The low-residence time conclusion inferred from the 'colour' attribute is in contrast with the results obtained for the contour 'roughness/smoothness', given that two-thirds of our LMPs exhibited a high degree of smoothness, suggesting in turn a relatively high degree of prermanence within the seawater medium. A number of studies (e.g. Doyle et al., 2011) have in fact associated smooth edges with older fragments that have been continuously polished by other particles or sediment. The relatively smooth nature of the LMP contours recorded in this study might also be the result of an abundance of primary LMPs in our sample, given that production pellets are generally characterised by a high degree of smoothness when compared to secondary LMPs. Further in-depth analysis of the LMPs through, for example, electron microscopy, would however be necessary to assess further the degradation and erosion aspects through an observation of the type (e.g. conchoidal, linear) of microscopic fractures visible within the same LMPs (Corcorant et al., 2009)

Given the time-consuming nature of the microplastic characterisation exercise through unaided human observation, a number of studies that investigate the robustness of alternative techniques for the same purpose, have been conducted to date. These include the one by Song et al. (2015), which ran a comparison between the values for a number of microplastic attributes (e.g. abundance, category, and dimensions) as determined through unaided human observation, stereomicroscopic examination and FT-IR (Fourier Transform Infrared) spectroscopy. This study concluded that, even though differences in the abundance of microplastic particles were reported through the different techniques, these were not statistically significant and that the FT-IR technique was the most accurate in reducing the missing and miscount rates.

Yet another related study (Primpke et al., 2017) coupled FTIR spectroscopy and image analyses, but the latter technique was not applied to 'raw' images of microplastic particles but rather to Focal Plane Array (FPA) $\mu \mathrm{FTIR}$ spectral datasets, allowing the automated interpretation of complex FTIR-imagery data. Another spectroscopy technique which can be coupled with microscopy is Raman spectroscopy. This is a 'surface technique' allowing for the identification of a broad range of size classes below $1 \mu \mathrm{m}$ in diameter (Cole et al., 2013). A limited number of previous studies (e.g. Pedrotti et al., 2016; Goldstein et al., 2011) have used in parallel different microplastics quantification techniques including unaided visual observation as well as scanning through a Zooscan device. However, these studies lacked any comprehensive comparative assessment about the performance of the different techniques used.

The contribution beyond the current state-of-the-art of marine environmental monitoring proposed by this study is along the same lines as that proposed by Deidun et al. (2018) for automated coastal litter monitoring by aerial drones. In fact, the high degree of robustness of the image analyses algorithm developed in the current study, as supported by the results of the comparative statistics obtained, suggests that the same methodology is at a high readiness level and next to uptake within the revised MSFD round of Descriptor 10 monitoring indicators (scheduled for release following the end of the first MSFD six-year cycle in 2018) as a routine and recognised protocol. The proposed image analysis protocol can, in addition, be incorporated in a beach LMP-recording citizen science campaign mediated, for example, through the use of smart phone applications coupled to good-quality photography. This would enable the delivery of automated LMP characterisation results to the general public directly in 
the field, if a simple but mandatory methodological protocol is specified (e.g. isolated LMPs are photographed against a clear background) and if its restricted to easily-extractable LMPs in the dry zone of beaches.

\section{Acknowledgements}

The authors would like to thank Prof. Victor Axiaq and his research team for providing further LMP samples for comparison purposes.

\section{Conflicts of interest}

None 


\section{References}

Abu-Hilal, A.H. and Al-Najjar, T.H., 2009. Plastic pellets on the beaches of the northern Gulf of Aqaba, Red Sea. Aquatic Ecosystem Health \& Management, 12(4), pp.461-470.

Axiak, V., Bezzina, M., Lomax, C., Deidun, A. and Edwards, K., 2017. First full investigation of levels of microplastics on sandy beaches in Malta. MEDCOAST.

Cole, M., Lindeque, P., Fileman, E., Halsband, C., Goodhead, R., Moger, J. and Galloway, T.S., 2013. Microplastic ingestion by zooplankton. Environmental science \& technology, 47(12), pp.66466655.

Corcoran, P.L., Biesinger, M.C. and Grifi, M., 2009. Plastics and beaches: a degrading relationship. Marine Pollution Bulletin, 58(1), pp.80-84.

Deidun, A., Gauci, A., Lagorio, S. and Galgani, F., 2018. Optimising beached litter monitoring protocols through aerial imagery. Marine pollution bulletin, 131, pp.212-217.

Deidun, A. and Schembri, P.J., 2004, October. Is faunal diversity on Maltese sandy beaches related to intensity of human use. In Proceedings of the First International Conference on the Management of Costal Recreational Resources-Beaches, Yacht Marinas and Coastal Ecotourism (pp. 20-23).

Doyle, M.J., Watson, W., Bowlin, N.M. and Sheavly, S.B., 2011. Plastic particles in coastal pelagic ecosystems of the Northeast Pacific Ocean. Marine Environmental Research, 71(1), pp.41-52.

Endo, S., Takizawa, R., Okuda, K., Takada, H., Chiba, K., Kanehiro, H., Ogi, H., Yamashita, R. and Date, T., 2005. Concentration of polychlorinated biphenyls (PCBs) in beached resin pellets: variability among individual particles and regional differences. Marine pollution bulletin, 50(10), pp.1103-1114.

Galgani, F., Hanke, G., Werner, S., Oosterbaan, L., Nilsson, P., Fleet, D., Kinsey, S., Thompson, R.C., Van Franeker, J., Vlachogianni, T. and Scoullos, M., 2013. Guidance on Monitoring of Marine Litter in European Seas. MSFD Technical Subgroup on Marine Litter (TSG-ML).

Gauci, A., Abela, J., Austad, M., Cassar, L.F. and Adami, K.Z., 2018. A Machine Learning approach for automatic land cover mapping from DSLR images over the Maltese Islands. Environmental modelling \& software, 99, pp.1-10.

Goldstein, M., Titmus, A., Witting, J., Engles, M. and Zettler, E., 2011. Eyeballs, nets, and digital scanners: the influence of methodology in assessing plastic debris in the North Pacific. 5th International Marine Debris Conference. UNEP and NOAA, poster 4.c.1.

Hidalgo-Ruz, V., Gutow, L., Thompson, R.C. and Thiel, M., 2012. Microplastics in the marine environment: a review of the methods used for identification and quantification. Environmental science \& technology, 46(6), pp.3060-3075.

Löder, M.G. and Gerdts, G., 2015. Methodology used for the detection and identification of microplastics - A critical appraisal. In Marine anthropogenic litter (pp. 201-227). Springer, Cham.

O'Neill, S.J. and Smith, N., 2014. Climate change and visual imagery. Wiley Interdisciplinary Reviews: Climate Change, 5(1), pp.73-87.

Pedrotti, M.L., Petit, S., Elineau, A., Bruzaud, S., Crebassa, J.C., Dumontet, B., Martí, E., Gorsky, G. and Cózar, A., 2016. Changes in the floating plastic pollution of the Mediterranean Sea in relation to the distance to land. PloS one, 11(8), p.e0161581.

Primpke, S., Lorenz, C., Rascher-Friesenhausen, R. and Gerdts, G., 2017. An automated approach for microplastics analysis using focal plane array (FPA) FTIR microscopy and image analysis. Analytical Methods, 9(9), pp.1499-1511. 
Setälä, O., Fleming-Lehtinen, V. and Lehtiniemi, M., 2014. Ingestion and transfer of microplastics in the planktonic food web. Environmental pollution, 185, pp.77-83.

Shiber, J.G., 1987. Plastic pellets and tar on Spain's Mediterranean beaches. Marine Pollution Bulletin, 18(2), pp.84-86.

Song, Y.K., Hong, S.H., Jang, M., Han, G.M., Rani, M., Lee, J. and Shim, W.J., 2015. A comparison of microscopic and spectroscopic identification methods for analysis of microplastics in environmental samples. Marine Pollution Bulletin, 93(1-2), pp.202-209.

Stolte, A., 2015. The detection of microplastics in beach sediments: extraction methods, biases, and results from samples along the German Baltic coast (Doctoral dissertation, Universität).

UNEP, 2014. Environment Programme at its first session. Proceedings of the United Nations Environment Assembly of the United Nations. 